\title{
Technology, culture and international stability
}

\author{
Larry Stapleton
}

Published online: 26 June 2013

(C) Springer-Verlag London 2013

Our world has never seemed so unstable and uncertain. If we are to survive and flourish as a species, it is becoming clear that we are entering a stage in human history in which we must engage with the complex systems of life itself.

The challenges that now lay before us are extremely complex and exist on a global scale. We must dramatically rethink many of the core assumptions we have taken for granted. We are fast discovering just how interconnected the universe is, and just how interdependent living and nonliving systems and sub-systems are upon each other.

Systems engineers close to these problems recognise the need for a radical new approach. For example, in the wake the Japanese environmental disaster of recent years, the systems engineering community represented by the International Federation of Automation and Control (IFAC) in Japan are recommending "glocal" thinking which delivers local science and engineering solutions with global vision. Humanity urgently needs integral, holistic, cross-disciplinary intellectual conversations and actions to create solutions that address the multi-dimensionality of the systems with which we must now engage.

It is perhaps surprising that, in the face of this growing awareness, modern science and engineering and the agencies which fund them remain entrenched in their traditional silos of activity. In the West, most of these activities are organised so as to resolve short-term, commercial concerns within the narrow agenda, that is, neo-liberalism. Our myopia will be judged harshly by our children's children's

L. Stapleton $(\bowtie)$

INSYTE Centre for Information Systems and Technoculture, Waterford Institute of Technology, Cork Road, Waterford, Ireland

e-mail: 1stapleton@wit.ie children. According to any reasonable moral framework, this will no longer do.

Technology proliferation contributes significantly to the systemic and structural effects we are now experiencing. The application of global systems of information, finance, energy and other resources has both negative and positive consequences. The recent financial crisis in the West is a testament to the fragility of our global systems and how little we really understand them. On the other hand, the global connectedness and the pluri-cultural context it has created present wonderful opportunities for our species to discover each other and learn how to care for this living system we call home.

The challenge for this special issue on technology, culture and international stability was to draw together strands of thought from across a range of disciplines which share a common concern with the role of science and engineering in systems of international stability. The current international stability intellectual discourse focuses upon climate change. We have chosen not to include contributions from climate change research and praxis (although you will find indirect contributions referring to environmental concerns). This is not because environmental systems are not important, but because we think they are well represented elsewhere.

In composing this issue, a range of contributions were sought which convey key dimensions of the problems we face but which have not received so much attention in the academic literature or popular press. Visionary contributions from the commercial sector, practical applicationsoriented research as well as deep theoretical reflections are included. This special issue also needed to draw from different cultures and academic subject domains. The hope is to inspire new conversations about the challenges we face, conversations which reach across the silos of science 
and the cultural boundaries whose walls seem, at times, very high indeed.

This issue of Artificial Intelligence and Society comprises a series of papers from members of the Technical Committee for Technology, Culture and International Stability (TC 9-5) of the International Federation of Automation and Control (IFAC). The committee comprises members from a wide range of disciplines who share a common interest in the problem of international stability. TC 9-5 is one of the oldest communities in IFAC, originating during the cold war period of the early 1980s. It is almost unique in the variety and range of members' interests and includes both academics and practitioners who regularly participate in its events and publications. All are invited to join us in our mission to improve international stability for all life on earth. Please contact the editor or the journal for details on how to get involved.

\section{Summary review of special issue contributions}

To appreciate our fluid, dynamic, international human system, we must seek out dimensions that are integral to that system and that are not so prone to fundamental change over relatively short periods of time. Human societies and their technologies are underpinned by and expressions of cultural values. Compared to other aspects of our international systems, human cultures and the values that underpin them are less prone to rapid change. Values can therefore offer deep insight into integral aspects of international human systems.

Technology and culture interpenetrate each other. Sismondo reminded us that modern technology is the "material culture" of science. Ihde described technology and culture as inseparable and coined the term "technoculture" to express this idea. A similar strand of thought can be traced back to cultural political scientists of the early 1990s (see Penley and Ross 1991 for example). In order to get under the surface of the cultural values which inform our international systems, a major theme tackled in this issue is "values".

We open with an industry perspective by David Martin, CEO of M-CAM Inc., Chair of Economic Innovation for the UN-Affiliated Intergovernmental Renewable Energy Organisation and founder of the Global Innovation Commons. It sets out the clear implications of the failure of Adam Smith's economic vision as a kind of control problem for international systems. Dr. Martin concludes that our current system of economic value is deeply flawed. He proposes an alternative value exchange system to our current financial model using an integral systems framework that is both holistic and inclusive of other forms of life besides humans.
In the next paper, Alan Cottey from Scientists for Global Responsibility notes that culturally embedded beliefs associated with human progress lead to an inherently unstable ecological system. This implies an inevitable radical overhaul of our current cultural value system if humanity is to survive. Professor Cottey proposes a radical scheme that institutes maxima to the legitimate incomes and assets of individuals. This scheme is the result of the necessary overhaul of current cultural beliefs about progress. He demonstrates how this can help avert the potential disaster likely under the current vision of human progress.

The next two papers explore the deep values which inform science and engineering praxis. The first is a contribution from Peter Carew and the editor, drawn from work at the INSYTE Centre in Ireland. The paper presents data gathered from an empirical study of technology development praxis. The data explore underlying cultural belief systems of technologists engaged in technology development and proliferation. It makes recommendations on how human-centred values can be fostered and engaged to enable greater empathy in contemporary system development and thereby strengthen international stability.

In her contribution, Marion Hersh discusses the limitations of engineering ethics as implemented in practice. Her paper explores how engineering and other activities are often carried out without any consideration of whether the activities are themselves ethical. Data are presented from a recent pilot study which investigates barriers to and enablers of ethical behaviour during the development of new technologies. Her work recognises the structural impediments to ethical behaviour in the engineering community and explores the relationship between individual and collective responsibility recognising the need for a support community, which enables engineers to think and behave ethically.

Vladimir Tsyganov of the Institute of Control Sciences at the Russian Academy of Sciences explores the tension between the limitations of global economic growth and a human psycho-physiology which seems to inculcate an ever growing desire for more consumption. He describes this as a control system problem that contributes to international instability. Professor Tsyganov concludes that the values of consumption, with their inherent growth limits, need to be replaced by a set of cultural values that do not have growth limits, such as those based upon creativity and spirituality.

International system stability is dependent upon sophisticated inter-networked systems of information sharing. This has raised significant challenges for technologists, including problems of privacy and message representation. Mihaly Heder's contribution notes that the possibility of interacting with remote services using 
natural language opens up new opportunities for sharing knowledge and could lead to, for example, more democratic access to government services. However, the question arises: "How can we best represent messages in such systems?" This paper argues that a text-based interface is best understood as an artificial agent that represents the interests of the remote party who is separated in time and space from the client. Technical and ethical considerations about the development of such an agent are discussed.

Sinead Somers' paper co-authored with the editor addresses inter-networked systems from an entirely different perspective. Throughout history and across cultures, farmers have been amongst the most innovative people in the world. Agriculture itself was a prehistoric system of innovation and technological development which transformed human society and our relationship with the living system in which we found ourselves. It is therefore surprising to find that agriculture lags behind other sectors in its uptake of new information technologies for the control and automation of farming systems. This is especially so in the developing world where these technologies have the potential to significantly improve conditions for farmers in poor communities. Policy has been too much focussed upon technological factors that are not immediately relevant to the existential concerns of farmers. Instead a glocal, human-centric approach is needed to ensure that local agricultural communities' voices are heard and that their embedded knowledge networks, institutions and cultural systems are carefully incorporated into e-agriculture policy interventions. A new framework of e-agriculture adoption and innovation is proposed which incorporates these dimensions of e-agriculture policy and so improves the e-readiness of farmers in less developed regions.

Twenty-first-century society is the most routinely and closely monitored in history, likened in previous $A I$ and Society issues to a "panopticon". Rusyaizila Ramli and Nasriah Zakaria from the School of Computer Sciences, Universiti Sains Malaysia, and from the College of Medicine, King Saud University, Saudi Arabia, consider the application of automatic behaviour monitoring of psychiatric in-patients. The particular focus of this paper is upon information privacy in such a system of care. They note that, for any new system like this to be successful, social and ethical issues must be prioritised over other considerations for the system. Mental health system providers must prioritise patients' privacy needs to avoid humiliation and prejudice. But how is this to be achieved? This paper applies a theoretical framework of privacy which appeared in a previous issue of $A I$ and Society. It demonstrates how this framework can inform the analysis and design process for an automatic behaviour monitoring system application in a psychiatric hospital.
The next two papers address policy development directed towards poor communities in Africa. Humancentred systems research has long advocated the need for systems designers to develop mechanisms by which all users' voices can be heard and incorporated in the systems development process. For example, critical theorists recommended that soft systems methodologies include both beneficiaries' and victims' voices during systems design (the so-called "BATWOVE" approach). External attitudes towards Africa can result in outsiders seeking to represent marginalised communities rather than trying to identify ways by which those communities might develop their own voice. The first of two papers explores the role of citizen journalism as a system by which conditions in the Kibera slum were improved. The "Voice of Kibera (VOK)" case study shows how a particular set of international development values resulted in residents taking a lead role in the production and consumption of news in one of the largest slums in the world. This in turn improved the lives of people in Kibera, contributing to better governance and more democratic systems and processes. In the second paper on Africa, Denis Dennehy and Mike FitzGibbon investigate the gap between international development policy and grass-roots practice south of the so-called developed countries of Europe and North America. Focussing upon non-governmental organisations (NGOs), they employ a knowledge management lens by which to both explore and address this gap.

Twenty-first-century enterprises need to establish flexible but trustworthy structures of doing business within a globally uncertain environment and, very often, in the midst of a complex web of inter-cultural interactions. The final group of papers look at these inter-cultural interactions from three different perspectives. The first paper by Luqi Wu and Michael Mahon presents a unique e-learning technology which applies computational intelligence to musical sounds in order to overcome cross-cultural language barriers and so stimulate inter-cultural understanding. The study demonstrates that a musical intelligence e-learning approach can benefit Chinese English-language students and has potential applications in other difficult cross-cultural language interactions. The second paper presents students' experiences of a European education programme for engineers. The programme was designed to inculcate an appreciation of inter-cultural interaction and help students come to terms with the chaotic reality of organisational life in the modern world. The paper provides suggestions on how to develop cross-disciplinary engineering and economics teaching which improves international trust and stability. In the third of this group of papers, Trevor Brown and Dietrich Brandt present empirical research which investigates the shifting influences on organisations as a result of the emergence of IT departments 
in high-growth economies. Using data gathered from over fifty interviews with IT managers, HR managers and expatriates, it demonstrates the organisational transformations resulting from the maturing of IT service industries in high-growth economies. It shows how a process of decentralisation in organisational structures is reinforced. Science and engineering professionals are challenged, move beyond simply "co-working" with other nationalities and ethnic groups and move towards a more collaborative, "open-hearted" approach to other cultural groups.

The special issue closes with the reflections of former IFAC president, Tibor Vamos, of the Hungarian Academy of Sciences. Professor Vamos draws upon the sweep of history to examine the role of human systems in the global information society. He challenges technologists to take their responsibility seriously by participating in the humanistic, broad anthropological search for improved conditions for life on earth. In doing so, they must take ownership of the effects of technological progress.

\section{Closing remarks and acknowledgements}

The papers which appear in this issue are not restricted to a systems science or engineering perspective. Neither are they limited to academic contributions alone. Instead, they are drawn from both industry and academia and from a variety of disciplines including ethics, engineering, information systems, international development and economics. The goal is to demonstrate the diversity of TC 9-5 and, hopefully, inspire the reader to examine new directions for research and practice which will improve conditions on the planet we call home.

The subjects covered in this special issue do not do justice to the work of my colleagues in IFAC. I have not included important developments in end-of-life management and recycling, mechatronics (such as the automated land mine clearance robots developed by Peter Kopacek's group in Vienna) or important work on the role of technology in the socio-economic recovery of post-conflict regions. Neither does this special issue touch on geographical information systems, low-cost automation, technology-driven transformation in less developed regions, environmental effects, human migration patterns, supplychain systems, e-healthcare architectures, global forecasting, global terrorism and criminal networks or microfinancial systems in Africa. All these and more are subjects covered in recent TC 9-5 conferences and symposia. However, we trust that the selection that is included demonstrates the breadth and depth of research on technology, culture and international stability.

A sincere thank you goes to the reviewers for their speedy turnaround of the papers, to the senior editorial team at AI and Society for their guidance and support and, especially, to the authors for their inspiring work.

It takes leadership, courage and humility for a wellestablished international journal to invite such a range of voices to speak in a single issue. I am deeply grateful that Artificial Intelligence and Society was willing to take that chance.

\section{Dedication}

This special issue is dedicated to the memory of Dr. Fred Kile. Dr. Kile was a former chairperson of TC 9-5 who passed away during the preparation of this issue. Over a period of 40 years, Fred tirelessly challenged science and engineering communities to create a more stable and sustainable world for the benefit of all life on Earth. His inspiration and vision will be sadly missed.

\section{References}

Penley C, Ross A (eds) (1991) Technoculture. University of Minnesota Press, Minneapolis 